LA-UR-01-2587

Approved for public release; distribution is unlimited.

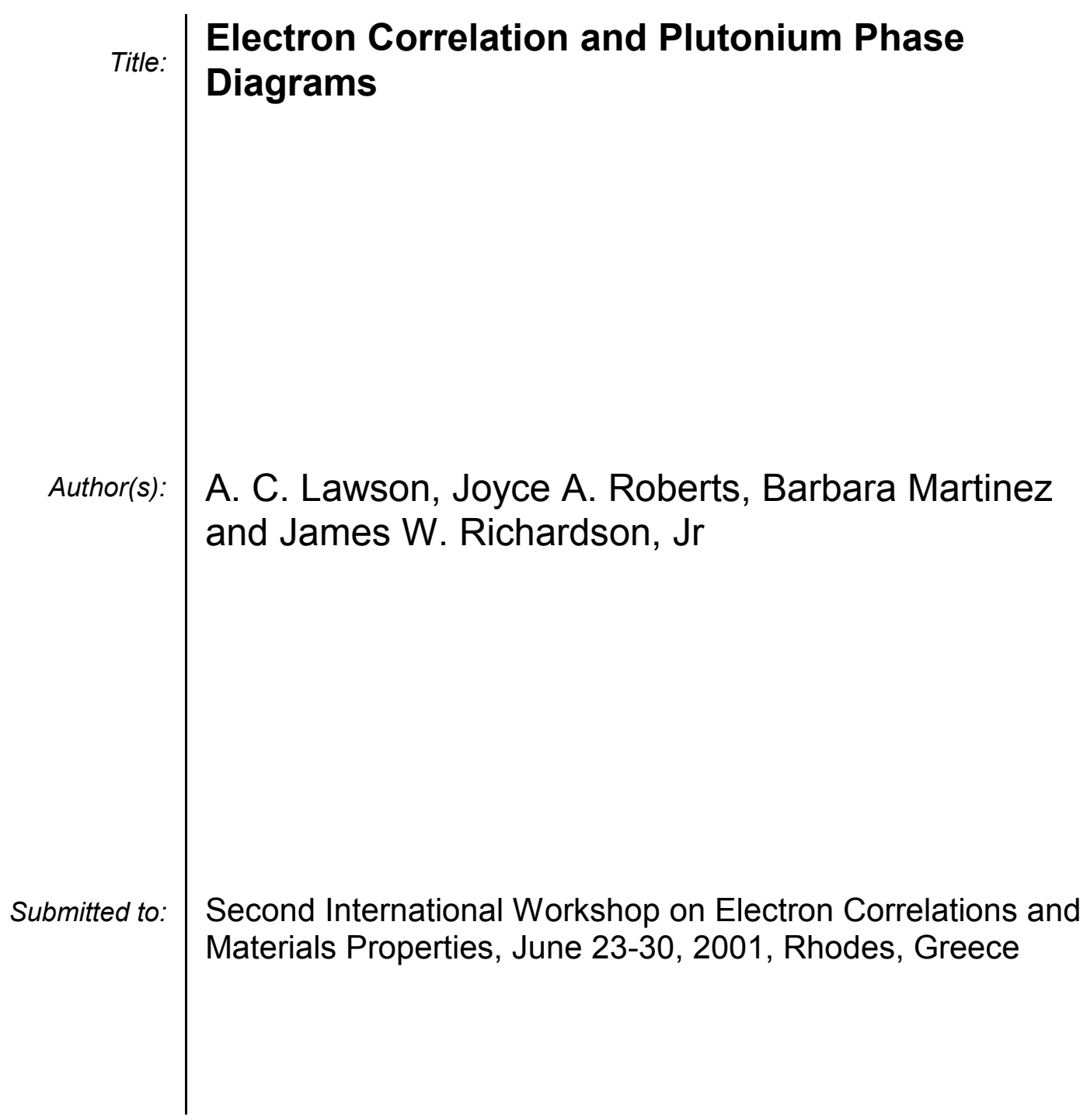

Los Alamos National Laboratory, an affirmative action/equal opportunity employer, is operated by the University of California for the U.S. Department of Energy under contract W-7405-ENG-36. By acceptance of this article, the publisher recognizes that the U.S. Government retains a nonexclusive, royaltyfree license to publish or reproduce the published form of this contribution, or to allow others to do so, for U.S. Government purposes. Los Alamos National Laboratory requests that the publisher identify this article as work performed under the auspices of the U.S. Department of Energy. Los Alamos National Laboratory strongly supports academic freedom and a researcher's right to publish; as an institution, however, the Laboratory does not endorse the viewpoint of a publication or guarantee its technical correctness. 


\title{
Electron Correlation and Plutonium Phase Diagrams
}

\author{
A. C. Lawson ", Joyce A. Roberts ${ }^{*}$, Barbara Martinez ${ }^{*}$ \\ and James W. Richardson, $\mathrm{Jr}^{\dagger}$
}

\section{INTRODUCTION}

Phase diagrams of plutonium metal are astonishingly complicated. The main feature is a sequence of crystallographically complex phases. Temperature, pressure and alloying shift the stability of these phases rapidly, so that pressure and alloy phase diagrams are also complicated. Another feature of the plutonium phase diagrams is the anomalously low melting point. How these two features, crystallographic complexity and low melting point, work to determine complicated phase diagrams is shown in Fig. 1. This figure, which is a composite of the relevant binary diagrams, shows that crystallographic complexity and low melting point are nearly exactly coincident. Such behavior is unique to the light actinides. It is natural to seek the source of this behavior in the collective properties of the $5 \mathrm{f}$ electrons, and in this paper we will trace some of the paths we are following in this search.

Fig. 2 shows the linear thermal expansion of unalloyed Pu metal. As the temperature is raised, there is a progression of phases from low symmetry to high symmetry as the melting point is reached. The $\alpha$ - and $\beta$-phases are monoclinic, the $\gamma$-phase is orthorhombic, the $\delta$-phase is FCC and the $\varepsilon$-phase is BCC. The $\delta^{\prime}$-phase is a transitional, tetragonal phase. A number of surprising features are apparent from this plot. First, the crystallographic complexity of the low temperature phases is unmatched by any other element: the $\alpha$ - and $\beta$-phases have 16 and 34 atoms per cell, respectively. This crystallographic complexity seems to be connected to the tendency of $\mathrm{Pu}$ to form intermetallic compounds with itself, the so-called "self-intermetallic" compounds

\footnotetext{
* Los Alamos National Laboratory, Los Alamos, NM 87545 USA

${ }^{\dagger}$ Argonne National Laboratory, Argonne, IL 60439 USA
} 


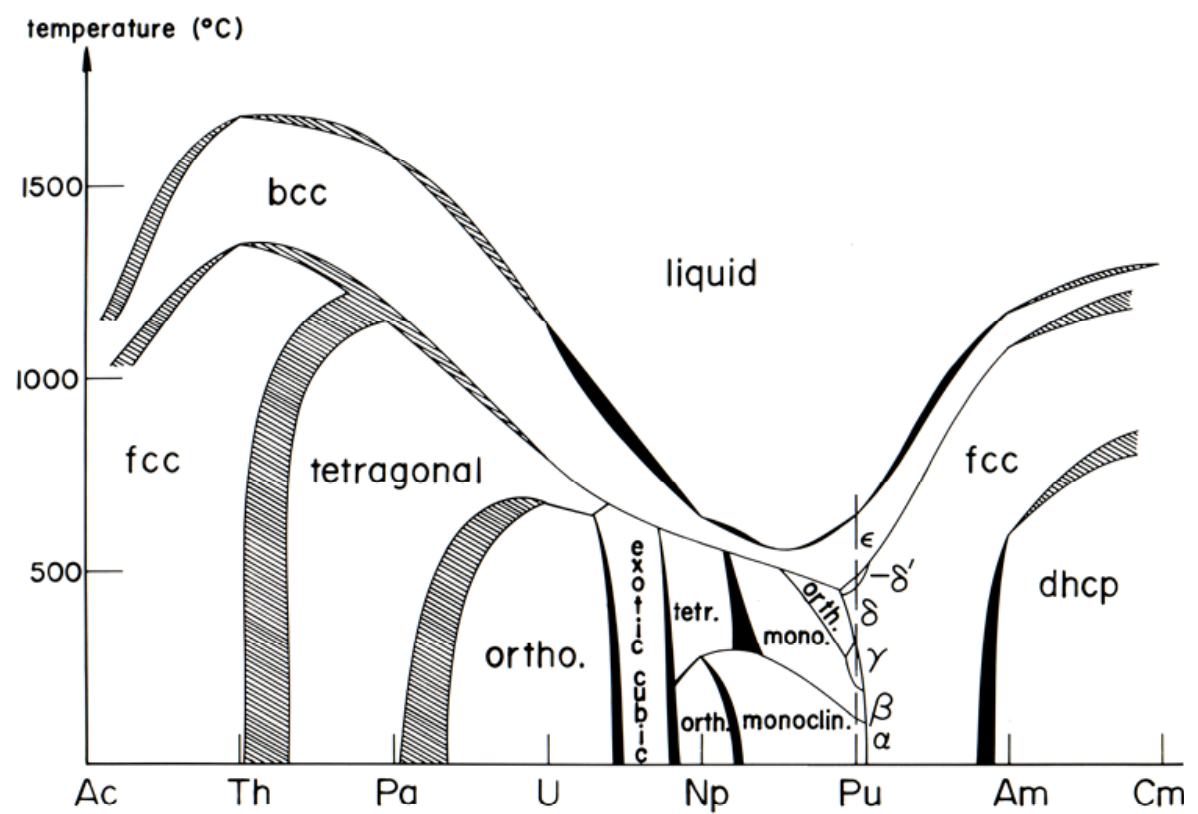

Figure 1. Composite phase diagram of the light actinides. Reprinted from J. Less-Common Metals, Vol. 90, J. L. Smith and E. A. Kmetko, Magnetism or bonding: a nearly periodic table of transition elements, pages 83-88, copyright 1983, with permission from Elsevier Science.

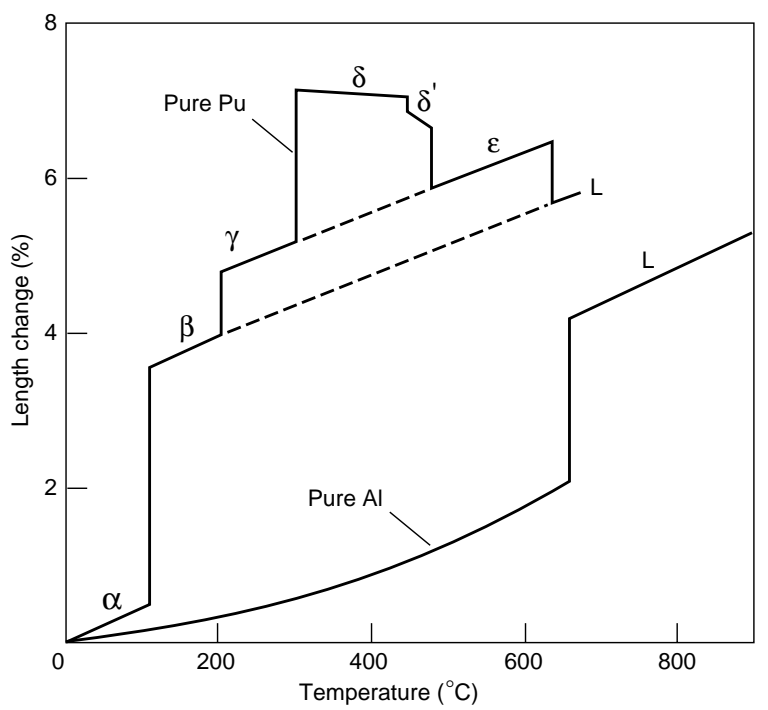

Figure 2. Linear thermal expansion of plutonium metal compared to that of aluminum. (Figure used by permission of Los Alamos Science.) 


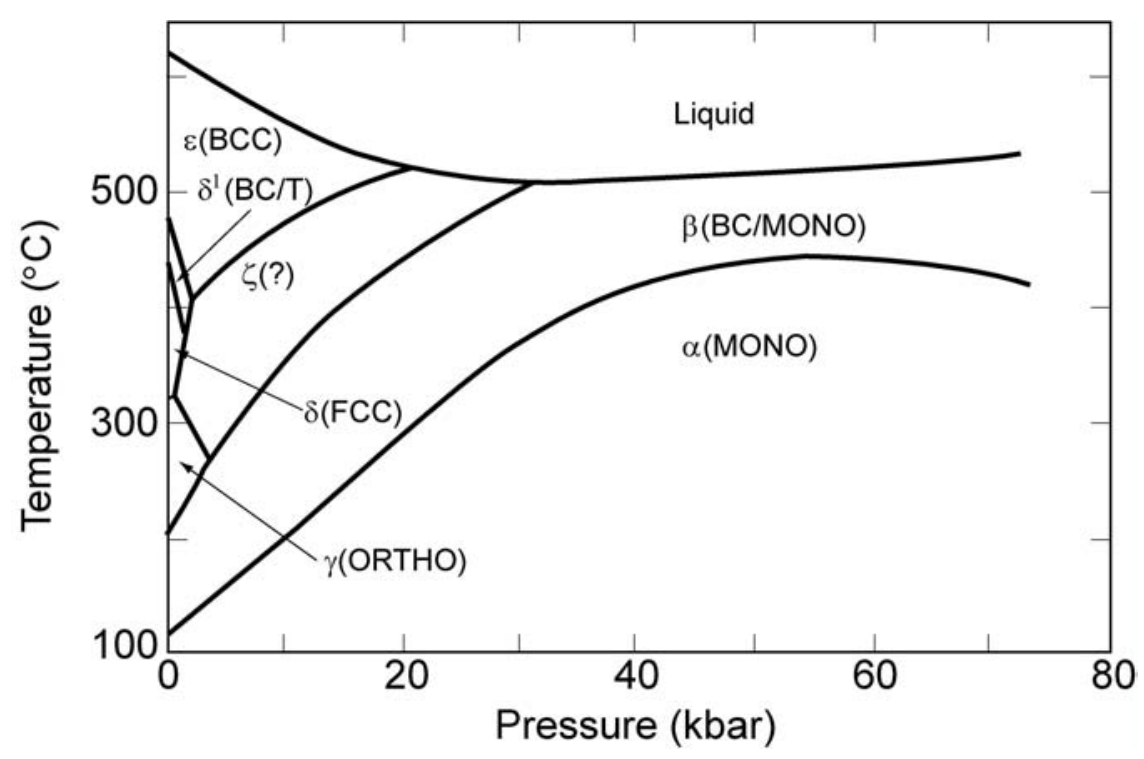

Figure 3. Pressure-temperature phase diagram of unalloyed Pu metal. Reprinted from J. Physics F., Vol. 6, E. A. Kmetko and H. H. Hill, Anomalous melting of f-electron metals (with attention to Pu), pages 1025-1037, copyright 1976, with permission of the Institute of Physics.

(Lawson et al., 1996) or to another, probably equivalent, tendency toward structural complexity in narrow band materials (Söderlind et al., 1995, Söderlind, 1998, Hecker, 2000, and Baskes, 2000). Second, the FCC $\delta$-phase has a surprising negative thermal expansion. Third, as noted, the melting point is surprisingly low, with a volume decrease at the melting point.

This multiplicity of crystallographically stable phases leads to complex phase diagrams, some of which will be displayed in the next section, and a few features of interest will be pointed out. The following section will discuss some aspects of disorder (or short range order) that may be expected in Pu phases. After that, we will discuss the special case of vibrational disorder and present some measurements of the Debye-Waller factors of the various phases in unalloyed $\mathrm{Pu}$. This leads to a discussion of the melting point of $\mathrm{Pu}$, based on the Lindemann rule. Finally, there is a discussion of the thermodynamics of $\mathrm{Pu}$, which leads us back to the topic of phase diagrams. At this point it is possible to make some contact with the topic of electron correlation.

\section{PU PHASE DIAGRAMS}

The pressure-temperature phase diagram of unalloyed $\mathrm{Pu}$, based on the work of Liptai and Friddle (1966) and modified by Kmetko and Hill (1976), is shown in Fig. 3 and reflects the complexity of the first two figures. An additional feature is the $\varsigma$-phase, stable only under pressure, whose structure is still undetermined. Notice that the melting point is further depressed by pressure to a minimum at $\sim 30 \mathrm{kbar}$, after which there is a gradual increase. There is an extremely suggestive analogy between the pressure phase 
diagram and the alloy phase diagrams that was originally put forward by Elliott. The idea is that alloying with elements that stabilize the $\delta$-phase ( $\mathrm{Ga}, \mathrm{Al}, \mathrm{Am}$, and some others) behaves like a negative pressure. This analogy is shown in Fig. 4. In this plot, the effect of alloying is shown with a phase diagram that is a composite of the Pu-Am (smaller solute) and Pu-Np (larger solute) diagrams. The Pu pressure phase diagram, shown in mirror image, matches the $\mathrm{Pu}-\mathrm{Np}$ diagram.

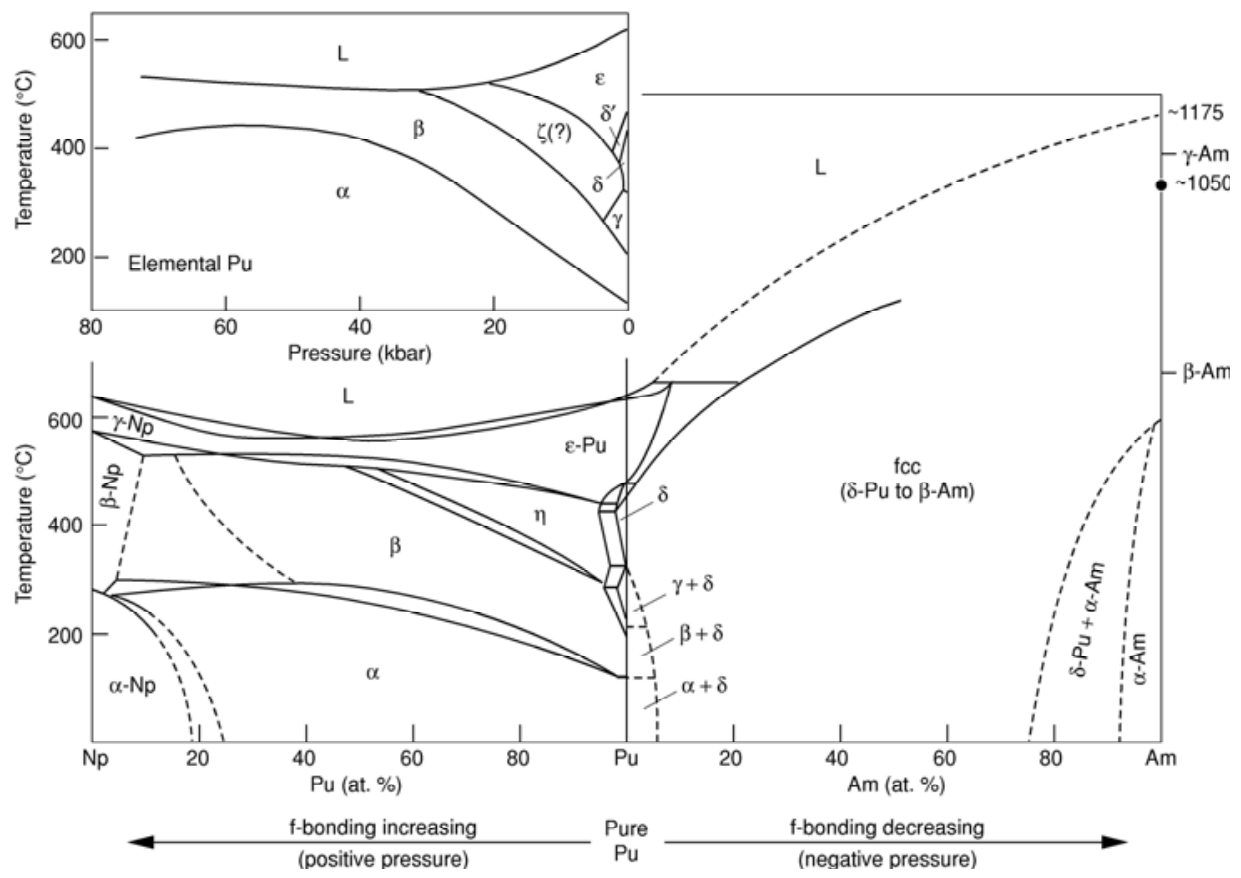

Figure 4. Composite alloy phase diagram for $\mathrm{Pu}-\mathrm{Np}$ and $\mathrm{Pu}-\mathrm{Am}$. The $\mathrm{Pu}-\mathrm{Np}$ bears a strong resemblance to the pressure-temperature diagram of Fig. 3, which is shown in mirror image. The Pu-Am diagram is similar to the $\mathrm{Pu}-\mathrm{Ga}$ diagram. (Figure used by permission of Los Alamos Science.)

The $\mathrm{Pu}-\mathrm{Ga}$ phase diagram is of technological importance, as a few percent $\mathrm{Ga}$ is found to stabilize the ductile FCC $\delta$-phase. The version of this diagram that was accepted in the US for a long time is presented in Fig. 5a and shows how the addition of Ga rapidly suppresses the low symmetry phases in favor of the ductile $\delta$-phase. Russian workers had believed that the $\delta$-phase alloy is thermodynamically unstable at low temperatures, and their diagram is shown in Fig. 5b. Hecker and Timofeeva (2000) tell the fascinating story of how consensus is being reached in a recent issue of Los Alamos Science. It has been extraordinarily difficult to reach agreement on the true, equilibrium phase diagram, as the equilibration times exceed the time-scale of ordinary laboratory experiments. For example, the eutectoid reaction in Fig. $5 \mathrm{~b}$ has never been observed in neutron diffraction experiments, which typically have a duration of a few days. 

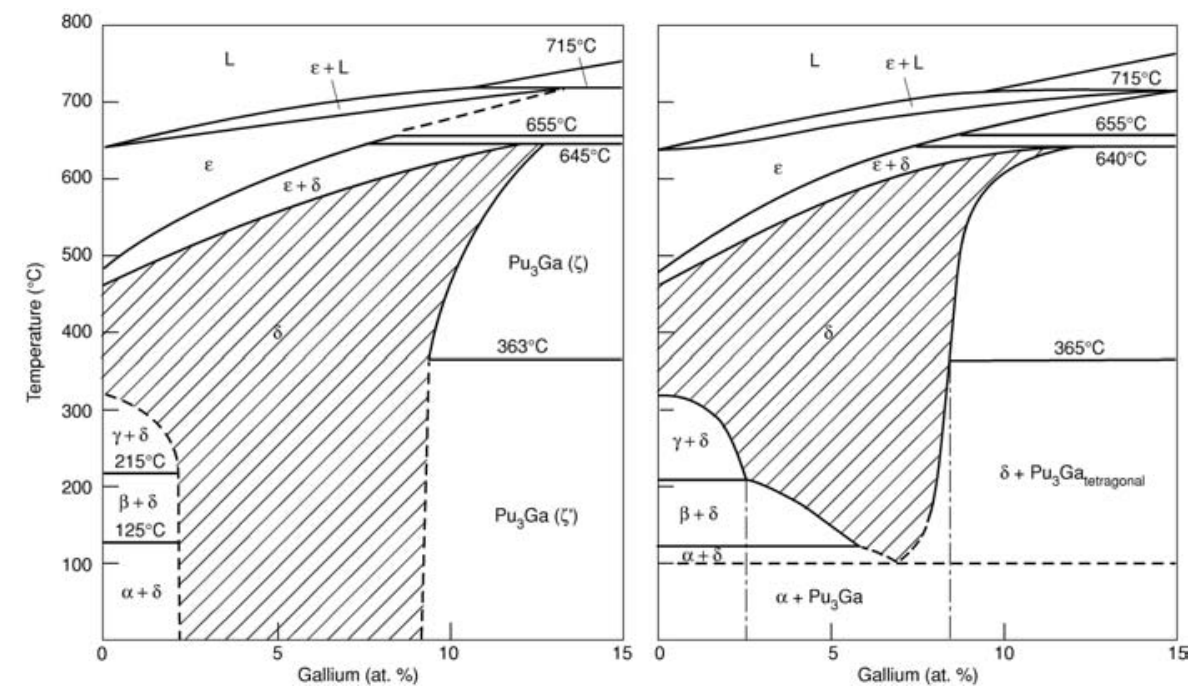

Figure 5. Alternative Pu-Ga alloy phase diagrams. The diagram at left (5a) indicates stability for $\mathrm{Pu}-2$ at. \% $\mathrm{Ga}$ at low temperatures, while the diagram at right $(5 \mathrm{~b})$ shows eutectoidal decomposition of the alloy. (Figure used by permission of Los Alamos Science.)

\section{PU DISORDER}

The structure of an ordered, crystalline material is correctly given by specifying the space group and atomic positions of the atoms. But perfectly ordered, crystalline materials do not exist in nature. Atomic positions may not be fully occupied, stoichiometric disorder or local atomic displacements may occur in alloys, and even in the purest materials, thermal vibrations will introduce deviations from perfect, periodic symmetry specified by the crystal space group. Deviations from perfect order are in principle observable as diffuse scattering. In practice, however, the diffuse scattering may be too small to be observable, even when the underlying disorder is large enough to be of theoretical significance. In addition one should heed the warning given by Welberry and Butler (1994) that the relationship between observed diffuse scattering and the underlying disorder is complicated and often counterintuitive.

As a specific example, let us consider an alloy $\mathrm{Pu}-2$ at. \% Ga. According to the phase diagrams that we have presented, this material contains enough gallium to exhibit the FCC crystal structure over a wide range of temperatures: from $450^{\circ} \mathrm{C}$ down to either the lowest temperatures (Fig. 5a) or at least to $100^{\circ} \mathrm{C}$ (Fig 5b). The structure of this alloy is usually presumed to be "disordered" FCC. This means that $2 \%$ of the Pu sites in the structure are occupied by gallium. Since the occupation is assumed to be random, the probability of occupation of any site by gallium is $2 \%$, independent of the occupation of neighboring sites. However, this assumption of independence need not be fulfilled exactly, as the Ga occupation of any site may influence the occupation of neighboring sites, and the occupation of gallium is then correlated. This correlation, described by the well-known Cowley order parameters, can be positive or negative, so that the gallium 
atoms may be thought to attract or repel each other, respectively (Warren, 1969). Whatever the state of correlation in $\mathrm{Pu}-2$ at. \% Ga, it is unobservable by x-ray or neutron diffraction, as the signal from only $2 \%$ solute is actually invisible in a practical experiment. (The classic example of the observation of such correlation is disordered $\mathrm{Cu}_{3} \mathrm{Au}$.) In fact, for neutron diffraction experiments discussed in this paper, the correlation is unobservable for an additional reason, namely that the neutron scattering cross sections for ${ }^{242} \mathrm{Pu}$ and for $\mathrm{Ga}$ are nearly identical.

In a Pu-Ga alloy, the $\mathrm{Ga}$ atoms are much smaller than the $\mathrm{Pu}$ atoms: the atomic radii are 1.4 and $1.6 \AA$, respectively. We can therefore anticipate that the structure will be disordered by the random displacement of the $\mathrm{Ga}$ atoms. If the $\mathrm{Ga}$ occupancies are correlated, as discussed in the previous paragraph, then the displacements might also be correlated, and diffuse scattering will be expected. However, in the case of Pu-2 at. $\% \mathrm{Ga}$, the expected scattering is again too small to be observed.

It is clear that crystal diffraction techniques are relatively insensitive to short-range order because the strength of the signal from the periodically ordered crystal swamps the relatively less intense diffuse scattering from the short-range order. The complementary EXAFS technique, which is sensitive to short-range order only, has been producing interesting results (Conradson, 2000) that are so far not completely understood.

\section{PU VIBRATIONS}

\subsection{Vibrational Correlations}

In every solid, the atoms vibrate. The motions of the atoms are given by the lattice dynamics, which can be calculated if the interaction between the atoms is known with sufficient accuracy. In favorable cases, the motions of the atoms can also be determined experimentally by inelastic neutron scattering. A case can be favorable if a single crystal is available; this is not currently the case for $\mathrm{Pu}-2$ at. \% Ga.

The existence of the atomic vibrations affects the result of a diffraction experiment in three ways. By far the largest effect is the attenuation of the Bragg reflections according to the Debye-Waller factor

$$
I=I_{0} e^{-k^{2}<u^{2}>}
$$

where $\left\langle\mathrm{u}^{2}\right\rangle$ is mean-square vibration amplitude of the atom, averaged over all atoms, all frequencies and all directions. $\left\langle\mathrm{u}^{2}\right\rangle$ can be calculated according to the Einstein model of lattice vibrations, which assumes that each atom vibrates independently of all the rest. This is a good model for the behavior of the Bragg intensities, and it is used routinely for the fitting of experimental diffraction data. The determination of $\left\langle\mathrm{u}^{2}\right\rangle$ vs. temperature data can be used to determine the Debye-Waller temperature, $\Theta_{\mathrm{DW}}$, as a function of temperature (Lawson et al., 2000a). $\Theta_{\text {DW }}$ is expected to be much more independent of temperature than is the heat capacity Debye temperature, $\Theta_{\mathrm{D}}$, because its moment of the phonon spectrum shifts between -1 and -2 between low and high temperatures, while that of $\Theta_{\mathrm{D}}$ shifts between -3 and +2 (Grimvall, 1999), and the second moment has hardly any temperature dependence at all at high temperatures (Lovesey, 1984). Experimentally, 
there is a good experimental correspondence between the values of $\Theta_{\mathrm{D}}$ and $\Theta_{\mathrm{DW}}$ measured at low temperatures (Lawson et al., 2000b)

But the atomic motions are in fact correlated, and this gives rise to diffuse scattering. The diffuse scattering can be calculated if a lattice dynamical model or measured phonon spectrum is available. If an exact calculation of the diffuse scattering is not possible, a good approximation is provided by the Debye model, which is based on a continuum approximation for the lattice dynamics. This turns out to be a good approximation to the correlation that is actually observed.

Fig. 6a shows a diffraction pattern of unalloyed $\delta^{\prime}-\mathrm{Pu}$ at $465^{\circ} \mathrm{C}$. The data have been fit by Rietveld analysis, and the tetragonal $\delta^{\prime}$ structure is confirmed. The error curve displayed below the data shows very clearly an oscillatory background due to vibrational correlations that have not been accounted for in the analysis. Fig. $6 \mathrm{~b}$ shows the same data fitted with the $\delta^{\prime}$ structure but with the background fitted with a series of oscillatory terms

$$
I(k)=\sum_{i=1}^{R_{\max }} A_{i} \frac{k^{2} \sin \left(k R_{i}\right)}{k R_{i}} .
$$

This function is an approximation to the thermal diffuse scattering for $\delta^{\prime}-\mathrm{Pu}$ at $465^{\circ} \mathrm{C}$. The $A_{i}$ 's, which are fit by Rietveld analysis, represent the correlation corrections to the Debye-Waller factor (which in turn represents the Einstein model).

The effect of vibrational correlations on the structure of a material can be represented by the pair distribution function, or PDF (Egami, 1998, McQueeney, 1998). The PDF is a one-dimensional plot of the density of atomic pairs in real space. Each peak in the PDF contains $\mathrm{N}(\mathrm{R})$ pairs at neighboring distance $\mathrm{R}$, given by the integral

$$
N(R)=\int_{p e a k} 4 \pi r^{2} \rho(r) d r .
$$

A "synthetic" PDF can be constructed from knowledge of the crystal structure. For each neighbor distance, one adds to the PDF a Gaussian term whose normalization gives the correct number of neighbors at that distance and whose width gives the vibrational correlation (including the autocorrelation). For each peak, the correlation can be found in one of three ways. At the lowest level of approximation, based on the Einstein model of independent atomic vibrations, one can use simply the width measured by the DebyeWaller factor. At this level of approximation, all the peaks in the PDF have the same width, which represents the autocorrelation. At the next level of approximation, the Debye model gives

$$
<u_{0} u_{0}>_{R}=<u^{2}>_{D W}\left(1-\frac{S i\left(k_{D} R\right)}{k_{D} R}\right)
$$

where $\mathrm{k}_{\mathrm{D}}$ is the Debye wavenumber, which depends only on the atomic density (Warren, 1967). The Debye model includes interatomic correlations calculated on a continuum model of the solid. A third, and even better level of approximation may be obtained by 
measuring the correlation corrections individually for each $\mathrm{R}$ by Rietveld analysis (Lawson, 2000b), as was done in Fig. 6. The Einstein approximation becomes exact for large enough R, e.g. $20 \AA$, as the interatomic correlations fall off with distance. In principle, the correlation atomic motions produce shifts in the peak positions of the PDF, and distortions of the Gaussian peak shape, but these are generally too small to be determined within the limits of resolution set by the Nyquist theorem, $\pi / \mathrm{Q}_{\max }$, where $\mathrm{Q}_{\max }$ is the maximum scattering wave number of the measurement, say about $25 \AA^{-1}$. The limitation to a Gaussian peak shape centered on fixed crystallographic distance could be relaxed with an extended model for the correlation corrected PDF.

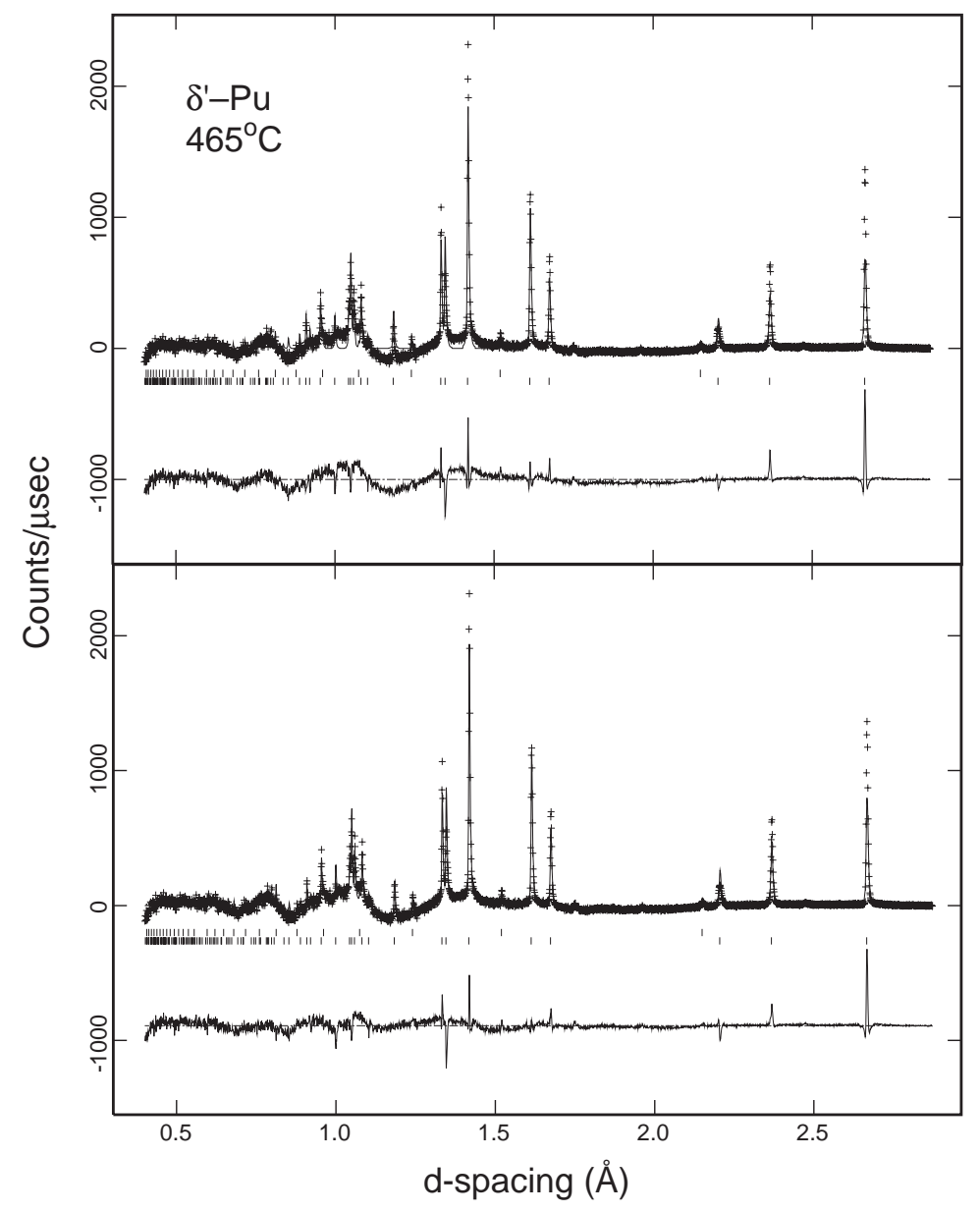

Figure 6. Rietveld fits of diffraction patterns for $\delta^{\prime}-\mathrm{Pu}$. The upper pattern (6a) is fit without correlation correction and the lower pattern (6b) includes only the first two terms of correlation correction. The crosses are the data, the line through the crosses is a fit to the data, and an error curve is shown below each pattern. Two sets of tic-marks indicate the allowed reflections for $\delta^{\prime}$-Pu (below) and for the vanadium radiological containment (above). 
An entirely different approach is to use Fourier transformation of the diffraction data to construct the entire PDF (Egami, 1998). This procedure gives the correct vibrational correlations, but places rather high demands on the quality of experimental data. As an example, consider the vibrations of $\mathrm{Pb}$ at two different temperatures, $300 \mathrm{~K}$ and $50 \mathrm{~K}$. Fig 7 shows the PDF constructed from the Einstein, Debye and correlation correction methods compared with the experimentally determined PDF obtained by Fourier transformation. As expected, the peaks of the PDFs at $300 \mathrm{~K}$ are broader, reflecting the larger thermal motion at that temperature. The various PDFs are generally in good agreement, except that the PDF based on the Einstein model overestimates the peak width at small R. The experimental PDF is noisier than usually obtained from this kind of work, because data were collected for only a few hours. Also we have not applied any corrections for sample container scattering, multiple scattering or absorption, or any Placzek corrections. The normalization of this PDF is based on the Reitveld scale factor and had to be adjusted upward by $20 \%$ to get the correct number of atoms under the PDF peaks (in other words, to agree with the PDFs obtained by other methods). The plot under the PDF curves shows the difference between the correlation corrected PDF and the Debye PDF. This difference is significant, but small.

Dimitrov et al. have proposed a method for extracting the phonon spectrum from powder diffraction data by matching the PDF obtained by Fourier transformation to that derived from a model phonon spectrum. Good agreement with experimentally determined phonon dispersion curves has been obtained for $\mathrm{Ni}$ and for $\mathrm{CaF}_{2}$. In view of the close agreement with the experimental PDFs for $\mathrm{Pb}$ (obtained either by Fourier transformation or by the correlation correction method) with the PDFs estimated by the Debye model, it would appear that experimental PDFs must be very accurate for this method to succeed. This is because the difference between a realistic phonon spectrum and one based on the Debye model (very unrealistic) must depend on the difference between experimental PDF and the synthetic PDF based on the Debye model. As we have shown, this latter difference is small.

As a further check on this point, we have compared the PDFs calculated from Monte Carlo simulations of $\mathrm{Al}$ and $\mathrm{Pb}$ under the assumption of nearest-neighbor forces. The nearest-neighbor model is not expected to produce an accurate prediction of the real metal, but it has the great advantage that the nearest neighbor force constants depend only on the single crystal elastic constants. Fig. 8 shows the anisotropic Young's modulus for $\mathrm{Al}, \mathrm{Ni}, \mathrm{Pb}$ and Ga-stabilized $\delta$-phase Pu. (For the first three, data are from Kittel, 1996, and for $\mathrm{Pu}$, from Ledbetter and Moment, 1976). $\mathrm{Pb}$ has a high elastic anisotropy, while $\mathrm{Al}$ is nearly isotropic. We can therefore investigate how the elastic anisotropy is reflected in the correlation correction that must be applied to the Einstein PDF. As shown in Fig. 9, the correlation correction for $\mathrm{Pb}$ is very similar to that of $\mathrm{Al}$, and both are similar to that expected from the Debye model. One concludes from these results that the PDFs must be measured and analyzed with extraordinary accuracy and that the results of the Dimitrov method may be highly susceptible to experimental error. Very recently (2001), Reichardt and Pintschovius have drawn similarly pessimistic conclusions.

It would be a considerable advantage to know the phonon spectra of the various phases of $\mathrm{Pu}$ so that the vibrational free energies can be calculated directly. PDF experiments on $\delta$-phase $\mathrm{Pu}$ have been undertaken in an attempt to get the phonon spectrum for this phase without the difficulty of growing a single crystal (Lashley et al., 2000). The discussion given here may argue in favor of growing a single crystal after all. 

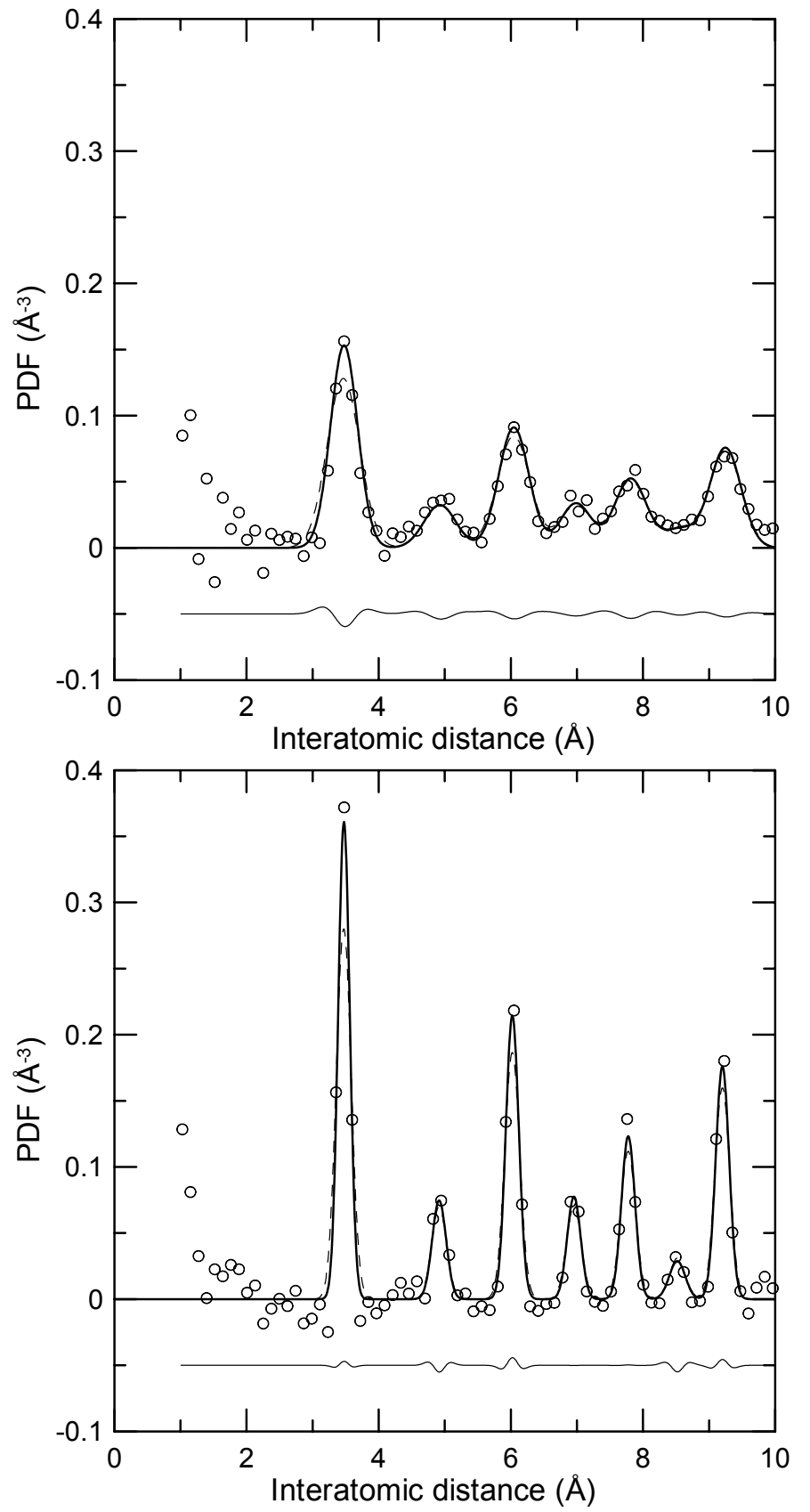

Figure 7. Pair Distribution Functions (PDFs) for lead at 300K (upper panel) and 50K (lower panel). The circles represent the PDF derived from Fourier transformation of the data. The solid line is the correlationcorrected PDF and the dashed line in the Einstein PDF. The curve under the full PDF is the difference between the Debye PDF and the correlation corrected PDF. 


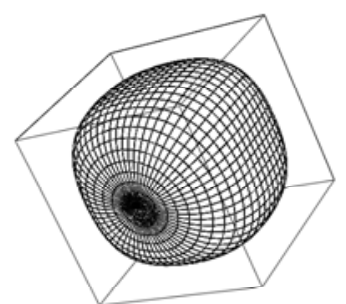

Aluminum

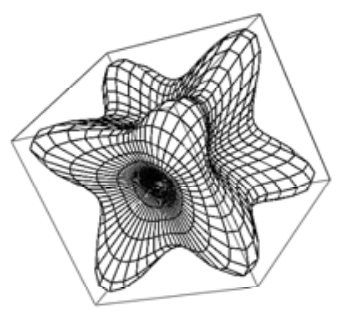

Lead

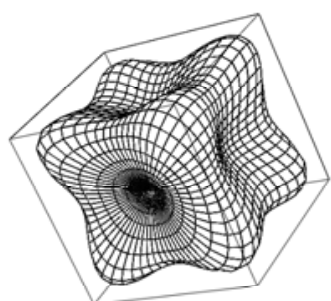

Nickel

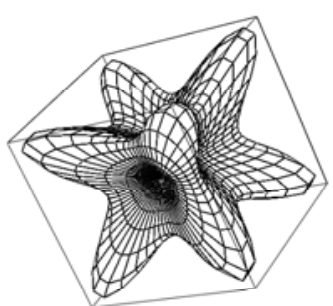

$\delta$-Plutonium

Figure 8. Young's modulus versus crystal direction for $\mathrm{Al}, \mathrm{Ni}, \mathrm{Pb}$ and $\delta$-phase $\mathrm{Pu}$. The scales are arbitrary.

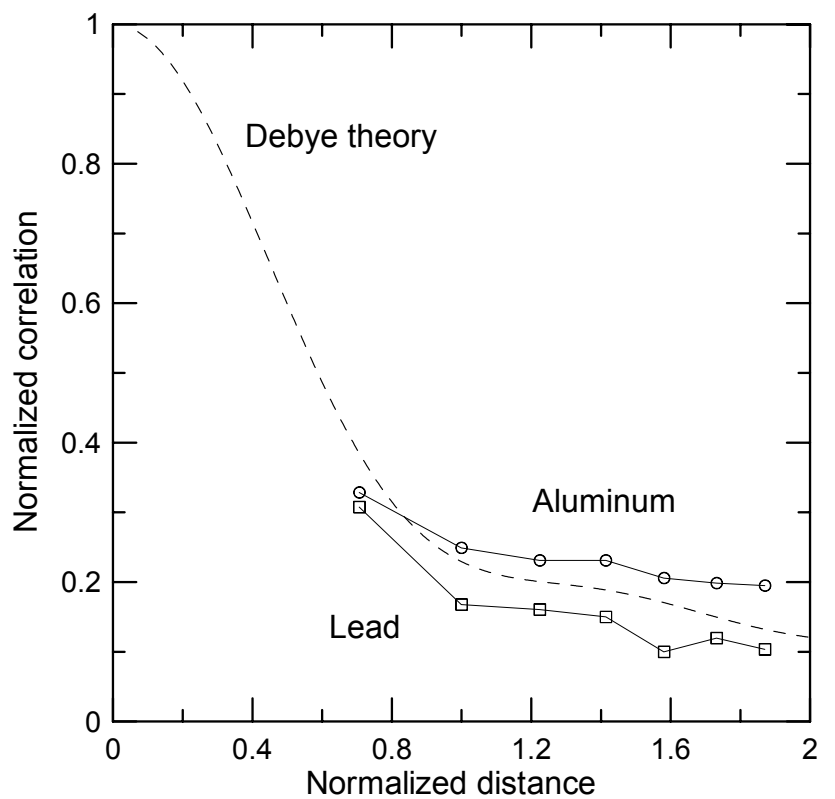

Figure 9. Normalized correlation $\left\langle\mathrm{u}_{0} \mathrm{u}_{\mathrm{n}}>/<\mathrm{u}_{0}{ }^{2}\right\rangle$ versus normalized distance $\left(\mathrm{R}_{\mathrm{n}} / \mathrm{a}_{0}\right)$ for two Monte Carlo models $(\mathrm{Pb}$ and $\mathrm{Al})$ and for the Debye model. 


\subsection{Debye-Waller Factors of Unalloyed Pu.}

Fig. 10 shows a plot of $\left\langle\mathrm{u}^{2}\right\rangle$ versus temperature for all the phases of unalloyed Pu. These data were obtained on the General Purpose Powder Diffractometer at IPNS ( $\mathrm{T} \geq 300 \mathrm{~K}$, Lawson et al., 1998, 1990) or on the High Intensity Powder Diffractometer at LANSCE ( $\mathrm{T}<300 \mathrm{~K}$, Lawson et al., 1992, 1993, Kwei et al., 1998). Because the temperature range of the data was small for most of the phases, we fit only $\Theta_{\mathrm{DW}}$ to the data, except for the $\alpha$-phase). The errors in the fitted $\Theta_{\mathrm{DW}} \mathrm{S}$ are less than $1 \mathrm{~K}$ for $\beta$ through $\varepsilon$-phases and about 10K for $\alpha$. (See Lawson et al., 1994, for details of the fitting process.) There is nothing in the $\left\langle\mathrm{u}^{2}>\right.$ curve that correlates with the negative thermal expansion of the $\delta$-phase. For each phase, the $\Theta_{\mathrm{DW}}$ is proportional to the inverse square root of the slope of the $<\mathrm{u}^{2}>$ vs. temperature curves, and the $\Theta_{\mathrm{DW}} \mathrm{s}$ are listed in Table 1 together with some values obtained from heat capacity and ultrasonic methods. The $\Theta_{\mathrm{DW}} \mathrm{S}$ are plotted in Fig. 11 together with a plot of $\Theta_{\mathrm{DW}}$ for Pu-2 at. \% Ga from Lawson et al. (2000a) for comparison. The $\Theta_{\mathrm{DW}} \mathrm{S}$ decrease with increasing temperature: such a trend is required for phase stability and is very similar to that in the alloy, except that there is a big jump between the $\Theta_{\mathrm{DW}}$ of $\alpha$ - and $\beta$ - Pu.

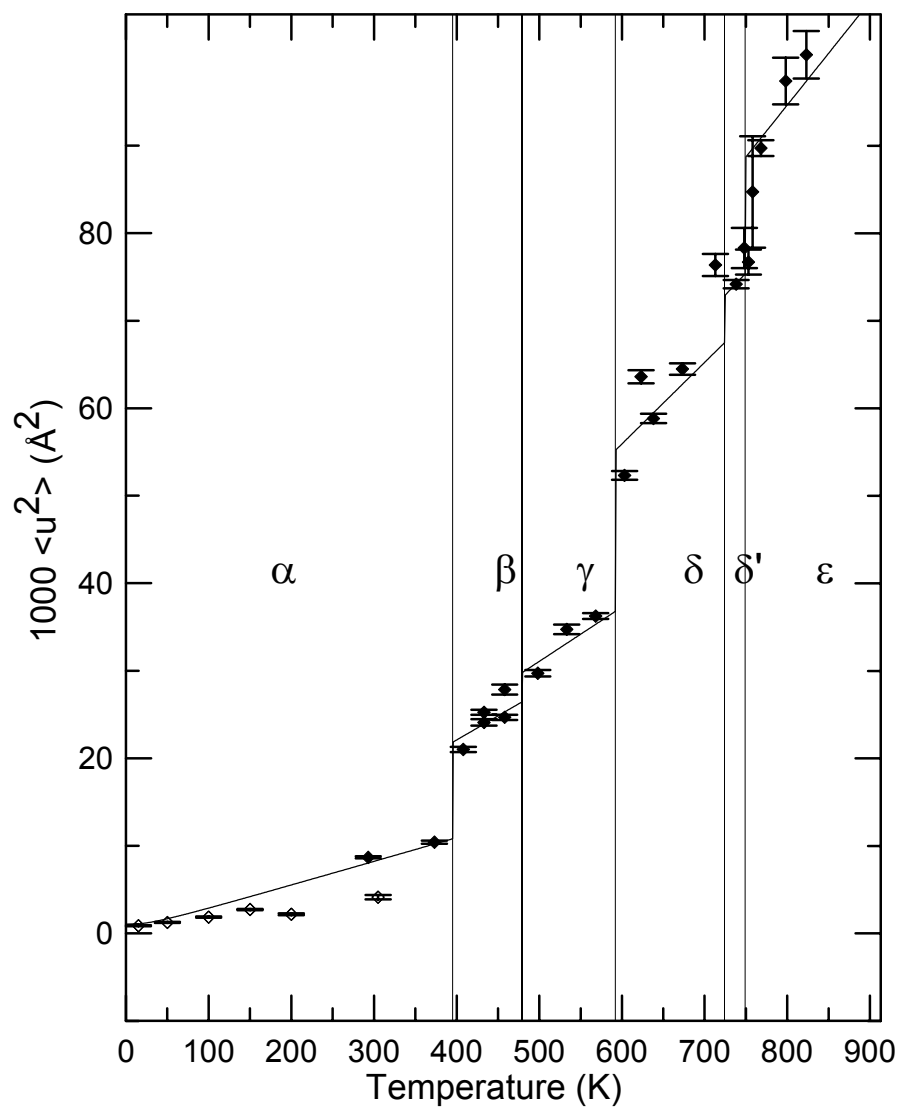

Figure 10. $<\mathrm{u}^{2}>$ versus temperature for all the phases of unalloyed plutonium metal. 


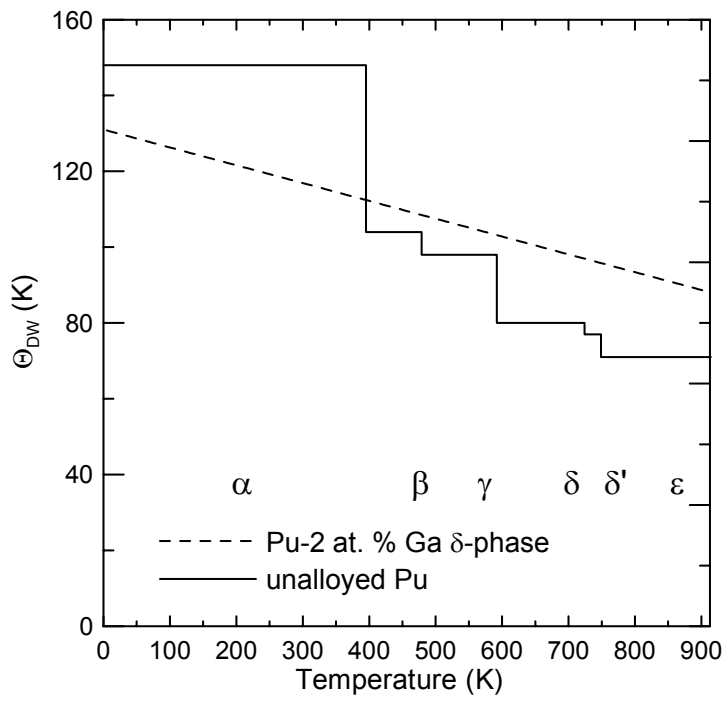

Figure 11. Debye-Waller temperatures for unalloyed $\mathrm{Pu}$ compared to those for $\mathrm{Pu}-2$ at. $\% \mathrm{Ga}$

TABLE 1. Values of $\Theta$ for the phases of Pu.

\begin{tabular}{|c|c|c|c|c|}
\hline phase & $\begin{array}{c}\Theta_{\mathrm{DW}}(\mathrm{K}) \\
\text { Debye-Waller }^{\mathrm{a}}\end{array}$ & $\begin{array}{c}\Theta_{\mathrm{D}}(\mathrm{K}) \\
\text { Heat Capacity }\end{array}$ & $\begin{array}{c}\Theta_{\text {Ultrasound }}(\mathrm{K}) \\
\text { Ultrasound }\end{array}$ & $\begin{array}{c}\Theta_{2}(\mathrm{~K}) \\
\text { Thermodynamic }^{\mathrm{g}}\end{array}$ \\
\hline$\alpha$ & 148 & $165^{c}$ & & 162 \\
\hline$\beta$ & 104 & & & 99.5 \\
\hline$\gamma$ & 98 & & & 94.1 \\
\hline$\delta$ & 80 & & & 92.2 \\
\hline$\delta^{\prime}$ & 77 & & & 84.9 \\
\hline$\varepsilon$ & 71 & & & 63.1 \\
\hline $\begin{array}{c}\delta \text {-phase } \\
\mathrm{Pu}-2 \text { at. } \% \mathrm{Ga}\end{array}$ & $121^{\mathrm{b}}$ & & & \\
\hline $\begin{array}{c}\delta \text {-phase } \\
\mathrm{Pu}-3 \text { at. } \% \mathrm{Ga} \\
\text { Single crvstal }\end{array}$ & & & $115^{\mathrm{e}}$ & \\
\hline $\begin{array}{c}\delta \text {-phase } \\
\mathrm{Pu}-5 \text { at. } \% \mathrm{Al}\end{array}$ & & & $132^{\mathrm{f}}$ & \\
\hline $\begin{array}{c}\delta \text {-phase } \\
\mathrm{Pu}-8 \text { at. } \% \mathrm{Al}\end{array}$ & & $130^{\mathrm{d}}$ & & \\
\hline $\begin{array}{l}\mathrm{a} \text { this work } \\
{ }^{\mathrm{b}} \text { Lawson et al. (20 } \\
{ }^{\mathrm{c}} \text { Lee et al. (1970) } \\
{ }^{\mathrm{d}} \text { Sandenaw (1960) } \\
{ }^{\mathrm{e}} \text { Ledbetter and M } \\
{ }^{\mathrm{f}} \text { Taylor et al. (196 } \\
{ }^{\mathrm{g}} \text { Wallace (1998) }\end{array}$ & nt (1976) & & & \\
\hline
\end{tabular}




\section{PU MELTING}

One of the many remarkable physical properties of plutonium metal is its low melting point, as shown in Fig. 1. A few years ago, we showed that this observation could be rationalized with the Lindemann melting rule because the elastic properties (the Debye-Waller temperature, $\Theta_{\mathrm{DW}}$ ) are strongly temperature dependent. The elastic stiffness decreases strongly with temperature as had been shown much earlier by ultrasonic measurements by Taylor et al. In fact, the $\Theta_{\mathrm{DW}} \mathrm{s}$ obtained by measurement of Debye-Waller factors are in quite good agreement with those obtained by ultrasonic measurement, as shown in Fig. 12.

The Lindemann rule states that a material melts when its vibrational amplitude is roughly equal to $10 \%$ of the interatomic distance. The vibrational amplitude at any temperature can be expressed in terms of the Debye temperature, $\Theta_{\mathrm{DW}}$. If $\Theta_{\mathrm{DW}}$ is temperature dependent, as it is for $\mathrm{Pu}$, it should be evaluated at the melting point. A convenient form of the Lindemann rule is

$$
T_{c}=\frac{M \Omega^{2 / 3} k_{B} \Theta_{m e l t}^{2} f^{2}}{3 \hbar^{2}}
$$

which suggests the plot of $\mathrm{T}_{\mathrm{m}} / \mathrm{M} \Omega^{2 / 3}$ vs $\Theta_{\text {melt }}{ }^{2}$ that is given in Fig. 13. This plot suggests the validity (with limited accuracy) of the Lindemenn rule. The trick is to evaluate $\Theta$ at the melting point, and we have used $\Theta=\Theta_{0}+\mathrm{cT}$, as suggested by Fig. 12, together with single crystal elastic data or Debye-Waller factor measurements. Then Eq. 5 has the melting point on both sides, but can nevertheless be solved explicitly, as shown by Lawson et al. (2000a, 2000c). The large (negative) value of c shown in Fig 14 gives a calculated value of the melting point that is in good agreement with the observed low melting point. In fact, the value of $-\mathrm{c}$ for $\mathrm{Pu}$ is one of the largest in the periodic table. (Details are given in Lawson, 2001.)

How can the atomic spring constants of $\mathrm{Pu}$ be temperature dependent? The usual explanation has to do with lattice anharmonicity. For an ordinary material, the thermal interatomic potential is asymmetric, and this asymmetry leads to thermal expansion. As this material is heated, the interatomic separation increases, and the vibrational frequencies decrease through the Grüneisen equation of state:

$$
\frac{\omega}{\omega_{0}}=\left[\frac{\Omega}{\Omega_{0}}\right]^{-\gamma_{G}}
$$

where $\gamma_{\mathrm{G}}$ is the Grüneisen constant and $\Omega$ is the atomic volume. $\gamma_{\mathrm{G}}$ 's are usually in the range of 1-3. A linear decrease of $\Theta_{\mathrm{DW}}$ with temperature is then a natural consequence of thermal expansion. But for $\mathrm{Pu}-2$ at. $\% \mathrm{Ga}$, the thermal expansion is very small, and the Grüneisen effect cannot be important here. Instead, it has been suggested that these materials undergo "harmonic softening" as the temperature increases, and this is assumed to be a purely electronic effect (Lawson et al., 2000a, Manley et al., 2001). According to this suggestion, the large values of $-\mathrm{c}$ for the light actinides arise - at least in part - from harmonic softening. 


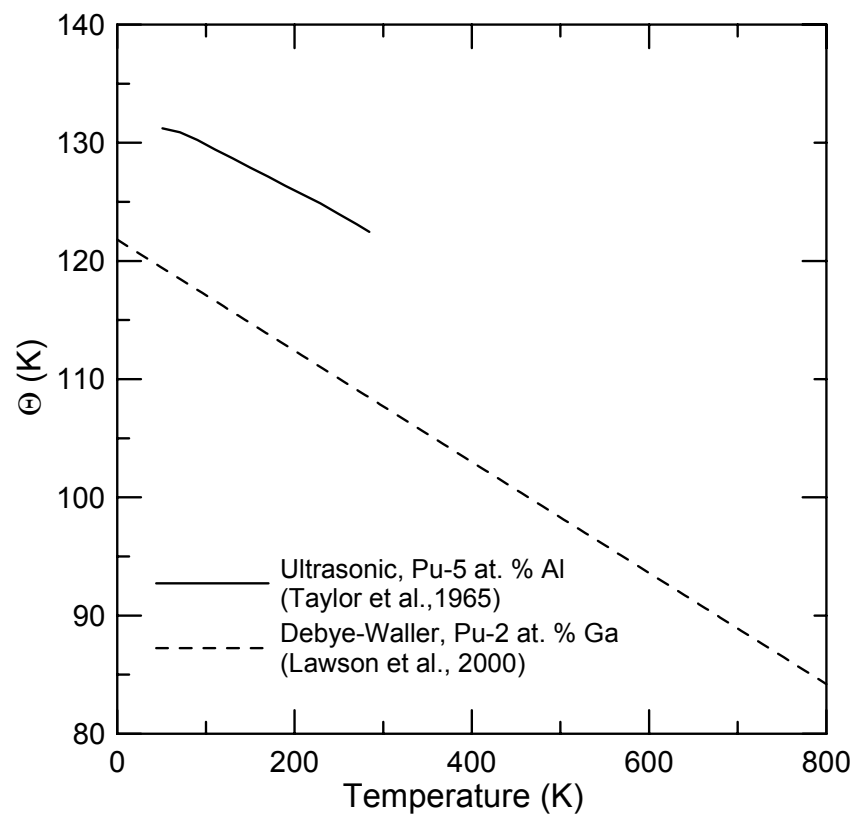

Figure 12. $\Theta_{\mathrm{DW}}$ and $\Theta_{\text {ultrasonic }}$ versus temperature for $\delta$-stabilized Pu alloys.

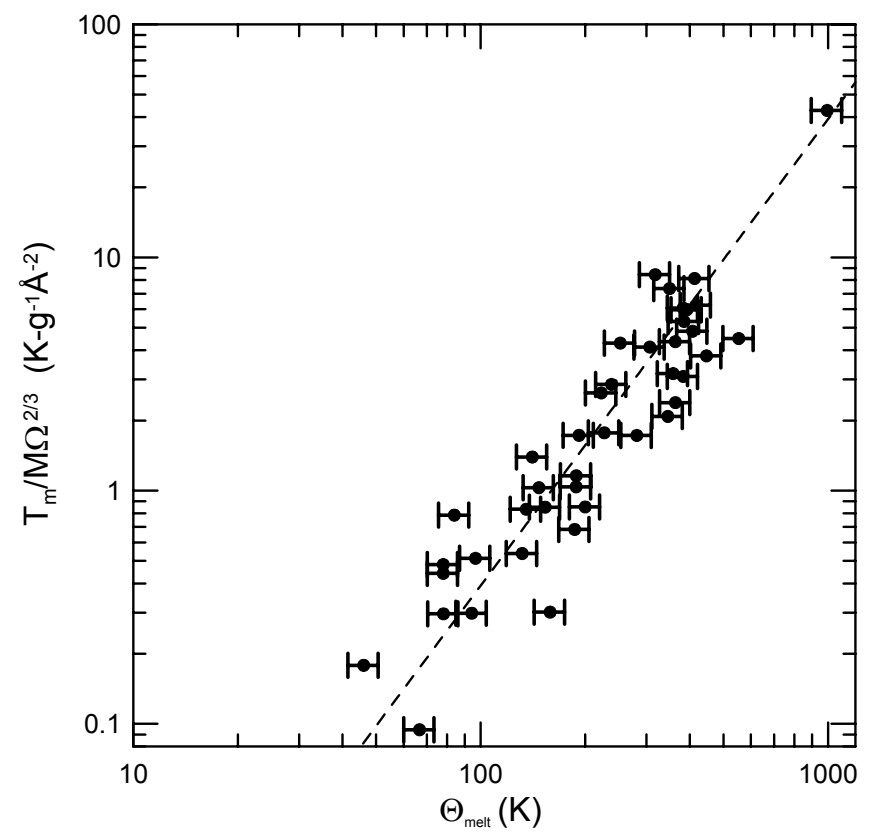

Figure 13. Normalized melting temperature $\left(\mathrm{T}_{\mathrm{m}} / \mathrm{M} \Omega^{2 / 3}\right)$ versus $\Theta_{\text {melt }}$, the Debye temperature at the melting point. $\Omega$ is the atomic volume and M is the atomic mass. Reprinted from Phil. Mag. B., 81:255 (2001), A. C. Lawson, An improved Lindemann melting rule, page 255, with permission from Taylor and Francis Limited, http://www.tandf.co.uk. 


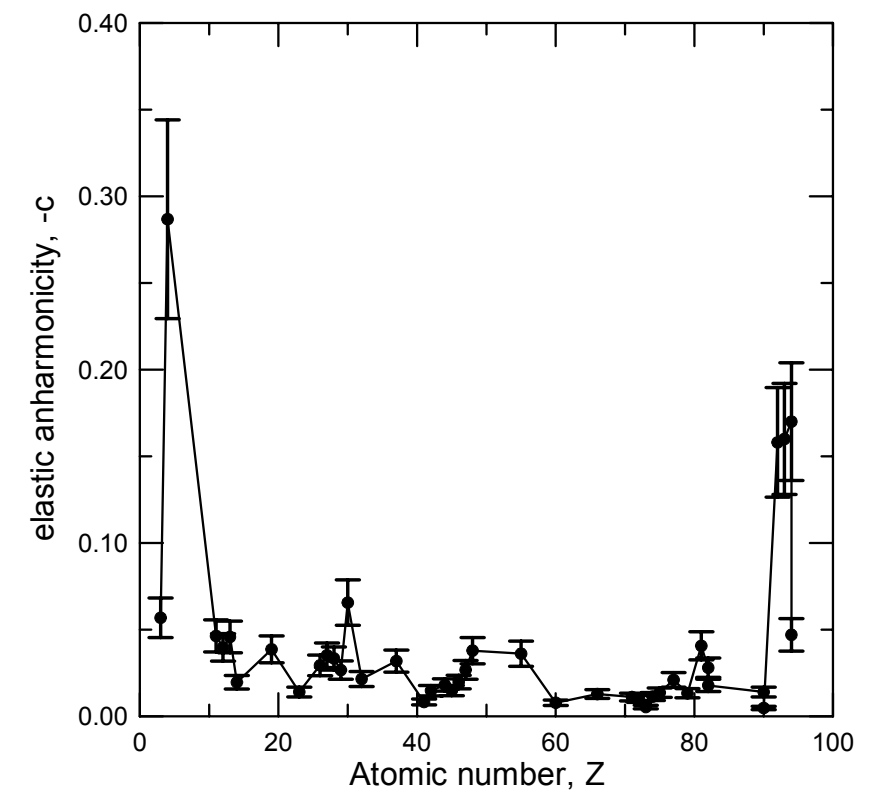

Figure 14. Elastic anharmonicity, $-\mathrm{c}$, defined by $\Theta=\Theta+\mathrm{cT}$, versus atomic number. Reprinted from Phil. Mag. B., 81:255 (2001), A. C. Lawson, An improved Lindemann melting rule, page 255, with permission from Taylor and Francis Limited, http://www.tandf.co.uk.

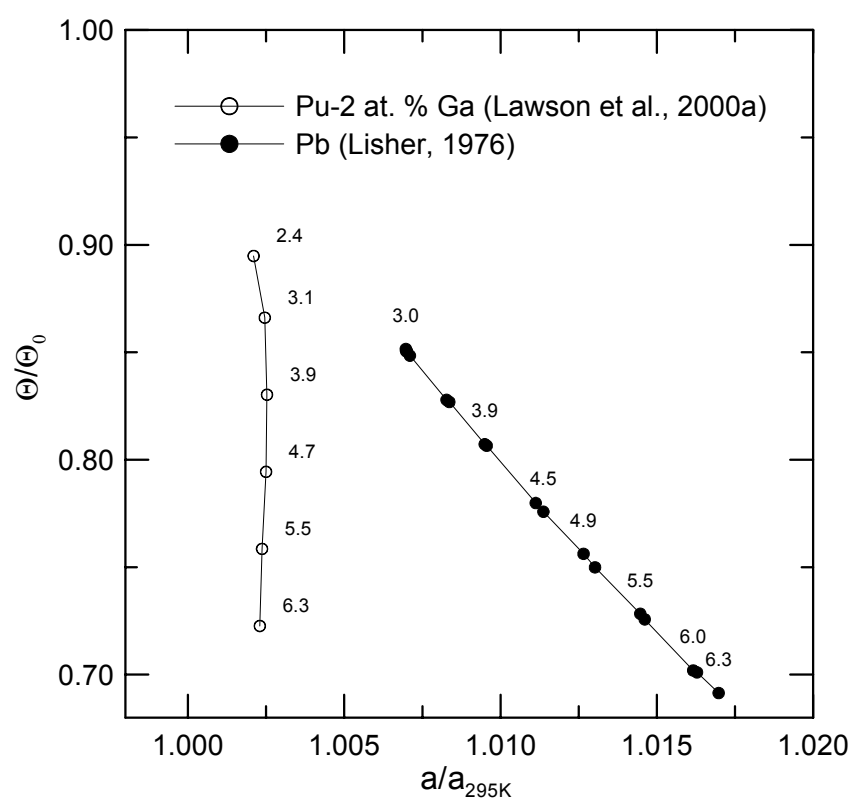

Figure 15. Normalized Debye temperature versus normalized lattice constant for $\mathrm{Pb}$ and $\delta$-phase $\mathrm{Pu}-2$ at. $\%$ Ga. The parameter is $\mathrm{T} / \Theta_{0}$. 
The difference between anharmonic and harmonic softening is illustrated in Fig. 15, which shows plots of normalized Debye temperature, $\Theta / \Theta_{0}$, versus the normalized lattice constant, $\mathrm{a} / \mathrm{a}_{295 \mathrm{~K}}$ for $\mathrm{Pb}$ and for Pu-2 at. \% Ga. The data are from Lisher, 1976, and Lawson et al., 2000a, respectively. For Grüneisen solids, such as $\mathrm{Pb}$, the strength of the atomic spring constants is mainly volume dependent, but for the light actinides is mainly temperature dependent. (The data in this figure could not be used for the evaluation of $\gamma_{\mathrm{G}}$, because the temperature is not constant.)

Manley et al. (Manley, 2000 and Manley et al., 2001) have observed harmonic softening in polycrystalline $\alpha-\mathrm{U}$ using inelastic neutron scattering over a wide range of temperatures. Their results show a continuous softening of the phonon spectrum in the orthorhombic $\alpha-\mathrm{U}$ phase as the temperature is increased.

\section{PU THERMODYNAMICS}

For $\Theta_{\mathrm{D}}$ independent of temperature, the vibrational heat capacity at constant volume is given by

$$
C_{v i b}=3 N k_{B}\left[12\left(T / \Theta_{D}\right)^{3} \int_{0}^{\Theta_{D} / T} \frac{u^{3} d u}{e^{\Theta_{D} / T}-1}+\frac{3 \Theta_{D} / T}{e^{\Theta_{D} / T}-1}\right]
$$

and this formula is modified to

$$
C_{\text {anharmonic }}=C_{v i b}\left(\frac{\Theta_{D, 0}}{\Theta_{D, 0}+c T}\right)^{2}
$$

for the case of $\Theta_{\mathrm{D}}=\Theta_{\mathrm{D}, 0}+\mathrm{cT}$. In these equations, $\Theta_{\mathrm{D}}$ is the Debye temperature, rather than the Debye-Waller temperature; these are respectively based on the +2 and -2 moments of the phonon spectrum (Grimvall, 1999). To these terms must be added the "dilatation" term

$$
C_{d i l}=9 \alpha^{2} \Omega B T
$$

and the electronic term, approximated by

$$
C_{e l}=\gamma_{e l} T .
$$

These models are evaluated and compared to the measured heat capacities in Fig. 16 for $\mathrm{Pb}, \alpha-\mathrm{Pu}, \delta-\mathrm{Pu}$ and $\alpha-\mathrm{U}$, materials for which either good or at least marginal data are available $(\mathrm{Pb}$ : Pankratz, 1982; $\alpha$-Pu: Lee et al., 1970; $\delta$-Pu: Sandenaw, 1960; $\alpha$-U: Holden, 1958). There is an extra heat capacity contribution for $\alpha-U$ at high temperatures, and this has been seen in three different measurements. This contribution has roughly the shape of a $\lambda$-anomaly, and the entropy under the heat capacity curve $\left(\mathrm{C}_{\mathrm{p}}-\mathrm{C}_{\mathrm{tot}}\right)$ is $\mathrm{R} \ln (1.6)$. 
This may be a measure of the lattice softening seen by Manley et al. Also unexplained are the enhanced values of $C_{p}$ in the $\beta$ - and $\gamma$-phases, and the fact that they are constant with temperature, rather than slightly increasing. Data for the heat capacity of $\delta$-phase $\mathrm{Pu}$ are marked by some kind of transient phenomenon - perhaps related to radiation damage - that has not received a satisfactory explanation. Better data are required for evaluation.

While the experimental situation is unclear for $\delta$-Pu, it is obvious from the general agreement of $C_{\text {tot }}$ with $C_{\text {exp }}$ that the temperature dependence of the heat capacity $\Theta_{D}$ for the two phases of $\mathrm{Pu}$ is much less than observed in the Debye-Waller factor $\Theta_{\mathrm{DW}}$. This apparent disagreement must be resolved by detailed measurements of the frequency dependence of the phonon spectra. Manley's work is a step in this direction.
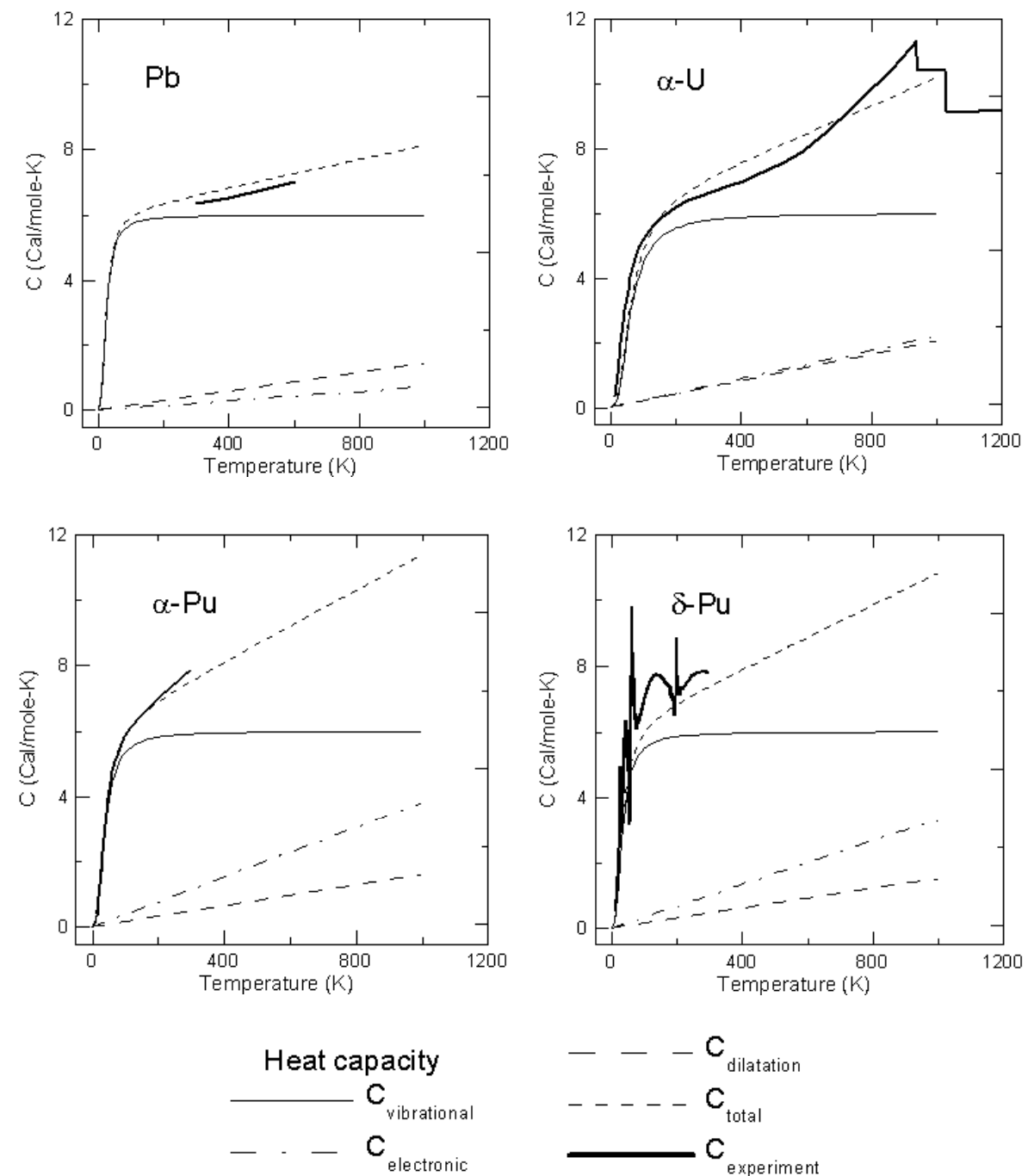

Figure 16. Heat capacities for $\mathrm{Pb}, \alpha-\mathrm{U}, \alpha-\mathrm{Pu}$ and $\delta$-phase $\mathrm{Pu}-8$ at. $\% \mathrm{Al}$. 
This becomes more than a textbook exercise when one considers all the uncertainties in actinide thermodynamics, and it may be a realistic way of determining the temperature dependence of the electronic term, which is certainly more complicated than Eq. (10) for the f-electron systems we are discussing.

Wallace (1998) has made careful estimates of the thermodynamic functions of all the phases of Pu based on available thermodynamic data. He finds it impossible to separate the "electronic" contributions from the "quasiharmonic" contributions, but the combined contributions from these terms is a significant part of the free energy. This is shown in Fig. 17, which shows the vibrational and electronic plus quasiharmonic contributions estimated by Wallace. It is clear that electron correlation terms could be important for phase stabilization, even if they make only a fractional contribution to the total energy.
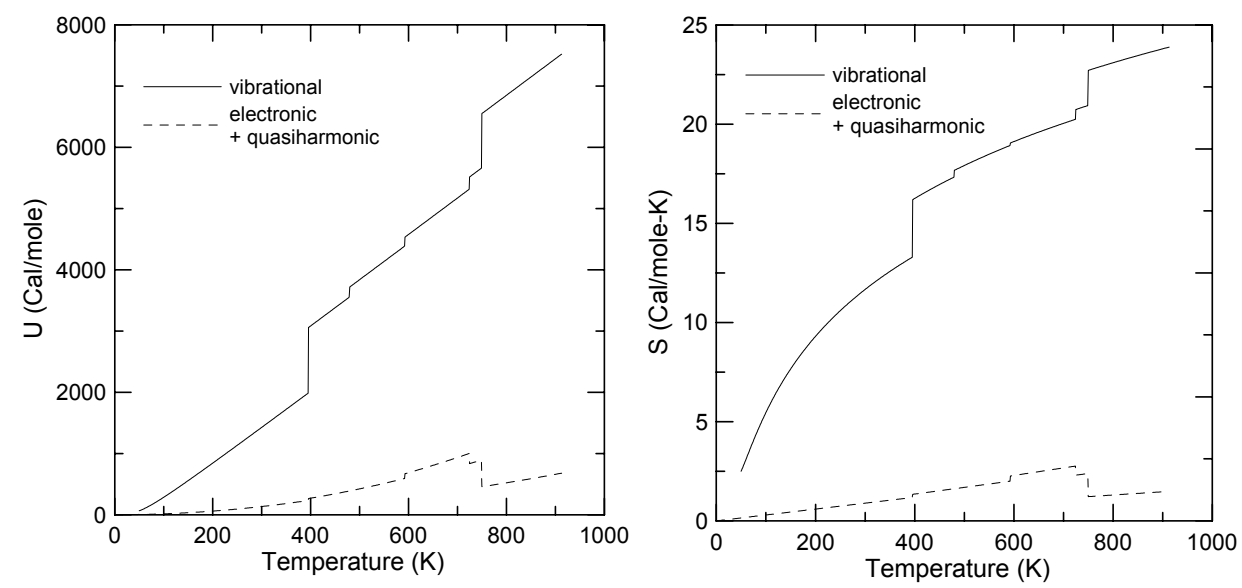

FIGURE 17. Internal energies (left) and entropies (right) for the phases of unalloyed $\mathrm{Pu}$

\section{CONCLUSIONS AND CONNECTIONS TO ELECTRON CORRELATION}

The energy and entropy curves for unalloyed Pu mark the extent of our progress toward an understanding of the phase diagrams presented at the beginning of the paper; it is clear that there is still a long way to go. It would be desirable to evaluate the vibrational thermodynamic functions from measured phonon spectra. However, this may require the growth of single crystals, if the PDF method using powder diffraction proves impractical for $\mathrm{Pu}$. The measurement of inelastic phonon densities of states from polycrystalline samples might be a practical method for all phases of Pu.

Estimates of the electronic contributions to the thermodynamics have been derived from the work of Wallace (1998). It would be of interest to compare these with theoretical estimates to evaluate the role of electron correlation in the phase stability of plutonium metal. As emphasized by Wallace, this will be an "enormous challenge" to electronic structure theory. In particular, we need a more predictive understanding of how narrow bands in Pu stabilize idiosyncratic crystal structures (Söderlind et al., 1995 and Söderlind, 1998). 
Finally we recall the role of electron entropy effects related to magnetism that shape the phase diagram of iron (Zener, 1967, and Kaufmann and Bernstein, 1970). We should be alert for similar effects in plutonium.

\section{ACKNOWLEDGMENTS}

We are pleased to thank Al Arko, Mike Baskes, David Becker, Mike Boring, Barry Cooper, Reed Elliott, Matthias Graf, Sig Hecker, Jason Lashley, Mike Manley, Rob McQueeney, Albert Migliori, Thomas Proffen, Mike Stevens, Stuart Trugman, and John Wills for very helpful discussions and we are grateful to Nikki Cooper, Sig Hecker and James L. Smith for use of their figures.

This work was supported under the auspices of the United States Department of Energy. The Manuel Lujan, Jr. Neutron Scattering Center is a National User Facility funded by the United States Department of Energy, Office of Basic Energy Science under contract No. W-7405-ENG-36. The Intense Pulsed Neutron Source is operated as a National User Facility by the United States Department of Energy, Office of Basic Energy Science - Materials Sciences, under contract No. W-31-109-ENG-38.

\section{REFERENCES}

Baskes, M. I., 2000, Atomistic model of plutonium, Phys. Rev. B. 62:15532.

Boring, A. Michael and Smith, James L., 2000, Plutonium condensed matter physics - a survey of theory and experiment, in Challenges In Plutonium Science, Necia Grant Cooper, ed., Los Alamos Science, No. 26, pp. 90-127; http://www.lanl.gov/external/science/lascience/.

Dmitrov, D. A., Louca, D., and Röder, H., 1999, Phonons from neutron powder diffraction, Phys. Rev. B 60:6204.

Conradson, Steven D., 2000, Where Is the Gallium? - Searching the plutonium lattice with XAFS, in Challenges in Plutonium Science, op. cit., pp. 356-363.

Egami, T., 1998, PDF analysis applied to crystalline materials, in Local Structure from Diffraction, S. L. J. Billinge and M. F. Thorpe, eds, Plenum, New York, pp. 1-21.

Grimvall, G., 1999, Thermophysical Properties of Materials, revised edition, Elsevier, Amsterdam, p. 89.

Hecker, Siegfried S. and Timofeeva, Lidia F., 2000, A tale of two diagrams, in Challenges in Plutonium Science, op. cit., pp. 244-251.

Hecker, Siegfried S., 2000, Plutonium and its alloys - from atoms to microstructure, in Challenges in Plutonium Science, op. cit., pp. 290-335.

Holden, A. N., 1958, Physical Metallurgy of Uranium, Addison-Wesley, Reading MA, p 47.

Kaufman, Larry and Bernstein, Harold, 1970, Computer Calculations of Phase Diagrams, Academic Press, New York, pp. 16-26.

Kittel, Charles, 1996, Introduction to Solid State Physics, $7^{\text {th }}$ Edition, New York, John Wiley and Sons, p. 91.

Kmetko, E. A., and Hill, H. H., 1976, Anomalous melting of f-electron metals (with attention to Pu), J. Phys. F. 6:1025.

Kwei, G. H., Louca, D., Billinge, S. J. L. and Rosenfeld, H. D., 1998, Recent local structure studies: metallic alloys, superconductors and proteins, in: Local Structure from Diffraction, op. cit., pp. 323-336.

Lashley, Jason C., Blau, Michael S., and Moment, Roger L., 2000, Preparing Single Crystals of GalliumStabilized Plutonium, in Challenges in Plutonium Science, op. cit., pp. 226-232.

Lawson, A. C., Cort, B., Goldstone, J. A., Wozniak, D., Hitterman, R. L. and Richardson, J. W., 1988, Diffraction studies of plutonium metal, IPNS Progress Report 1986-1988, Argonne National Laboratory, p. 50 .

Lawson, A. C., Goldstone, J. A., Vaninaetti, J., Eash, D., Cort, B., Hitternan, R., and Faber, J., 1990, Neutron diffraction study of $\delta^{\prime}$ and $\varepsilon$-plutonium, IPNS Progress Report 1988-1990, Argonne National Laboratory, p 28. 
Lawson, A. C., Goldstone, J. A., Cort, B. and Diebolt, L., 1992, Debye-Waller factors of $\alpha$-plutonium metal, LANSCE Experiment Reports - 1991 Run Cycle, Los Alamos National Laboratory, LA-12407-PR, p 33.

Lawson, A. C., Goldstone, J. A., Cort, B. and Von Dreele, R. B., 1993, Debye-Waller Factors of $\alpha$-plutonium, LANSCE Experiment Reports - 1992 Run Cycle, Los Alamos National Laboratory, LA-12647-PR, p 46.

Lawson, A. C., Goldstone, J. A., Cort, B., Sheldon, R. I. and Foltyn, E. M., 1994, Debye-Waller factors of the light actinide elements, in: Actinide Processing: Methods and Materials, B. Mishra and W. A. Averill, eds., TMS. Warrendale PA, pp. 31-43.

Lawson, A., C., Goldstone, J., A., Cort, Martinez, R., J., Vigil, F., A., Zocco, T. G., Richardson, J. W., Jr., and Mueller, M. H., 1996, Structure of $\zeta$-phase Plutonium-Uranium, Acta Cryst. B 52:32.

Lawson, A. C., Martinez, B., Roberts, J. A., Bennett, B. I., and Richardson, J. W., Jr., 2000a, Melting of the light actinides, Phil. Mag. B 80:53.

Lawson, A. C., Martinez, B., Von Dreele, R. B., Roberts, J. A., Sheldon, R. I. and Richardson, J. W., Jr., 2000b, Vibrational order in $\mathrm{Pu}_{0.98} \mathrm{Ga}_{0.02}$, Phil. Mag. B 80:1169.

Lawson, Andrew C., Martinez, Barbara, Roberts Joyce A., Richardson, James W., Jr., and Bennett, Bard I., 2000c, Atomic vibrations and melting in plutonium, in Challenges in Plutonium Science, op. cit., pp. 190201.

Lawson, A. C., 2001, An improved Lindemann melting rule, Phil Mag B 81:255.

Ledbetter, H. M. and Moment, R. L., 1976, Elastic properties of face-centered-cubic plutonium, Acta Met. 24:891.

Lee, J. A., Mendelssohn, K., and Sutcliffe, P. W., 1970, Specific heats of plutonium and neptunium, Proc. Roy. Soc. Lond. A. 317:303.

Liptai, R. G., and Friddle, R. J., 1966, The phase diagram of plutonium at pressures up to 75 kbar, J. LessCommon Metals 10:292.

Lisher, E., J., 1976, The Debye-Waller factors of lead from 296 to 550K, Acta Cryst. A 32:506.

Lovesey, S. W., 1984, Theory of Neutron Scattering from Condensed Matter, Vol. I, Oxford University Press, Oxford, pp. 112-113.

Manley, M. E., Fultz, B., McQueeney, R. J., Brown, C. M., Hults, W. L, Smith, J. L., Thoma, D. J., Osborn, R and Robertson, J. L., 2001, Large Harmonic Softening of the Phonon Density of States of Uranium, Phys. Rev. Lett. 86:3076.

Manley, Michael E., 2000, Vibrational softening in $\alpha$-uranium, in Challenges in Plutonium Science, op. cit., pp. 202-207.

McQueeney, R. J., 1998, Dynamic radial distribution function from inelastic neutron scattering, Phys. Rev. B 57:10560.

Pankratz, L. B., 1982, Thermodynamic Properties of Elements and Oxides, U.S. Dept. of the Interior, Bureau of Mines, Washington, p. 294.

Reichardt, W., and Pintschovius, L., 2001, Influence of phonons of the pair distribution function deduced from neutron powder diffraction, Phys. Rev. B 63:174302.

Sandenaw, T. A., 1960, Heat capacity, thermal expansion and electrical resistivity of an 8 a/o aluminumplutonium (delta-phase stabilized) alloy below 300K, J. Phys. Chem. Solids 16:329.

Smith, J. L., and Kmetko, E. A., 1983, Magnetism or bonding: a nearly periodic table of transition elements, J. Less-Common Metals 90:83.

Söderlind, Per, Eriksson, Olle, Johannson, Börje, Willis, J. M. and Boring, A.M. 1995, A unified picture of the crystal structures of metals, Nature 374:524.

Söderlind, P., 1998, Theory of the crystal structures of cerium and the light actinides, Advances in Physics 47:959.

Taylor, J. C., Loasby, R., G., Dean, J. D., and Linford, P. F., 1965, Some physical properties of plutonium at low temperatures, in: Plutonium 1965, A. E. Kay and M. B. Waldron, eds., Chapman and Hall, London, pp 162-175.

Wallace, D. C., 1998, Electronic and phonon properties of six crystalline phases of Pu metal, Phys. Rev. B 58:15433.

Warren, B. E., 1969, X-Ray Diffraction, Addison Wesley, Reading, MA, reprint:, Dover Publications,. Mineola, NY.

Welberry, T. R., and Butler, B. D., 1994, Interpretation of diffuse x-ray scattering via models of disorder, J. Appl. Cryst. 27:205.

Wills, John M., and Eriksson, Olle, 2000, Electronic structure of $\alpha$ - and $\delta$-plutonium, in Challenges in Plutonium Science, op. cit., pp 129-151.

Zener, Clarence, 1967, Influence of entropy in phase stabilization, in Phase Stability in Metals an Alloys, Rudman, Peter S., Stringer, John, and Jaffee, Robert I., eds, McGraw-Hill, New York, pp. 25-38. 